\title{
Pleiotropic Functions of FoxN1: Regulating Different Target Genes during Embryogenesis and Nymph Molting in the Brown Planthopper
}

\author{
Yu-Xuan Ye ${ }^{1(\mathbb{D})}$ and Chuan-Xi Zhang ${ }^{1,2, *(1)}$ \\ 1 Institute of Insect Science, Zhejiang University, Hangzhou 310058, China; yeyuxuan@zju.edu.cn \\ 2 State Key Laboratory for Managing Biotic and Chemical Threats to the Quality and Safety of Agro-Products, \\ Institute of Plant Virology, Ningbo University, Ningbo 315211, China \\ * Correspondence: chxzhang@zju.edu.cn
}

Received: 21 April 2020; Accepted: 12 June 2020; Published: 13 June 2020

\begin{abstract}
FoxN1 gene belongs to the forkhead box gene family that comprises a diverse group of "winged helix" transcription factors that have been implicated in a variety of biochemical and cellular processes. In the brown planthopper (BPH), FoxN1 is highly expressed in the ovaries and newly laid eggs, where it acted as an indispensable gene through its molecular targets to regulate early embryonic development. Moreover, the results of the RNAi experiments indicated that Nilaparvata lugens FoxN1 (NlFoxN1) exhibited pleiotropism: they not only affected the embryogenesis, but also played an important role in molting. RNA-seq and RNAi were further used to reveal potential target genes of NlFoxN1 in different stages. In the eggs, ten downregulated genes were defined as potential target genes of NlFoxN1 because of the similar expression patterns and functions with NlFoxN1. Knockdown of NlFoxN1 or any of these genes prevented the development of the eggs, resulting in a zero hatchability. In the nymphs, NlFoxN1 regulated the expression of a keratin gene, type I cytoskeletal keratin 9 (NlKrt9), to participate in the formation of an intermediate filament framework. Depletion of NlFoxN1 or NlKrt9 in nymphs, BPHs failed to shed their old cuticle during nymph-to-nymph or nymph-to-adult molting and the mortality was almost $100 \%$. Altogether, the pleiotropic roles of NlFoxN1 during embryogenesis and nymph molting were supported by the ability to coordinate the temporal and spatial gene expression of their target genes.
\end{abstract}

Keywords: FoxN1; brown planthopper; pleiotropism; embryogenesis; keratin gene

\section{Introduction}

FoxN1 gene belongs to the forkhead-box (Fox) gene family that encodes a large family of transcription factors characterized by a "winged-helix" DNA-binding domain [1]. Forkhead domain (FHD) is very well conserved across the Fox family and across various eukaryotic species; it extends about 100 amino acids in length [2].

FoxN1 was first well characterized both in mouse and humans, and designated as a nude gene [3]. FoxN1 is a transcription factor specific to epithelial cells and known to regulate the differentiation of several tissues [4]. FoxN1 expression could be detectable in the entire skin, particularly interfollicular epidermis and hair follicle, including developing whisker pads, nail primordial, hair follicles of eyebrows, and the epidermis of mouth, nose, ears, and tail [5]. In the skin, FoxN1 is mainly expressed in the keratinocytes of the first suprabasal layer and is a key transcription factor involved in the regulation of keratinocytes growth and differentiation [6,7]. The lack of functional FoxN1 protein leads to a nude phenotype in mice and humans, which is characterized by the lack of visible hair, and skin and nail abnormalities [8]. FoxN1 gene expression in the skin, as also seen in the thymus [9], mutations in the 
"nude" FoxN1 gene induce the hairless phenotype, associated with a severe combined immunodeficiency (SCID) phenotype [10]. During embryogenesis, FoxN1 is essential for the development of the thymus, the primary lymphoid organ that supports T-cell development and selection [11]. Similarly to the mouse Foxn1 gene, the human FOXN1 gene contains eight coding exons and two different first exons (not contributing to the protein sequence) that undergo alternative splicing [7]. Exon 1a and exon $1 \mathrm{~b}$ are under the control of two different promoters, thereby affording FOXN1 tissue specificity; the promoter 1a is active both in thymus and skin and the promoter $1 \mathrm{~b}$ only in the skin [12].

The pleiotropic roles of Fox proteins during the embryonic development and homeostasis of adult tissues is supported by the ability to coordinate the temporal and spatial gene expression of their target genes [13]. However, the molecular targets regulated by FoxN1 in different tissues or developmental stages to exert different functions remain to be elucidated.

Because of the absence of thymus organs, the expression and function of FoxN1 in insects might be different from those in mammals. To better understand the pleiotropic mechanism of FoxN1 and its potential application in insect pest control, we used the brown planthopper (BPH), Nilaparvata lugens Stål (Hemiptera: Delphacidae), one of the most destructive insect pests of rice crops [14], as a model to explore the functions and the potential transcriptional targets of FoxN1 in insects.

\section{Results}

\subsection{Sequence Analysis and Expression Patterns of NlFoxN1}

There are two duplicated FoxN1 genes in the N. lugens genome (Figure 1a). NlFoxN1a and NlFoxN1b share the same number of exons, the same cDNA sequences, and differ only in intron length. Similarly to the mouse and human FoxN1 genes, NlFoxN1 contains eight coding exons [12]. The cDNA of NlFoxN1 was amplified, cloned, and sequenced. The complete ORF sequence of NlFoxN1 is $1941 \mathrm{bp}$ long. BLAST analysis of the 111-amino-acid DNA binding domain of NlFoxN1 in NCBI revealed its homology with Papilio xuthus Forkhead box protein N1 with a percentage of identity of $75 \%$. Multiple sequence alignments were performed among FoxN1 orthologs from 11 species using the ClustalX program, and the result showed that the sequences of FoxN1 were highly conserved in vertebrates and invertebrates (Figure 1b).

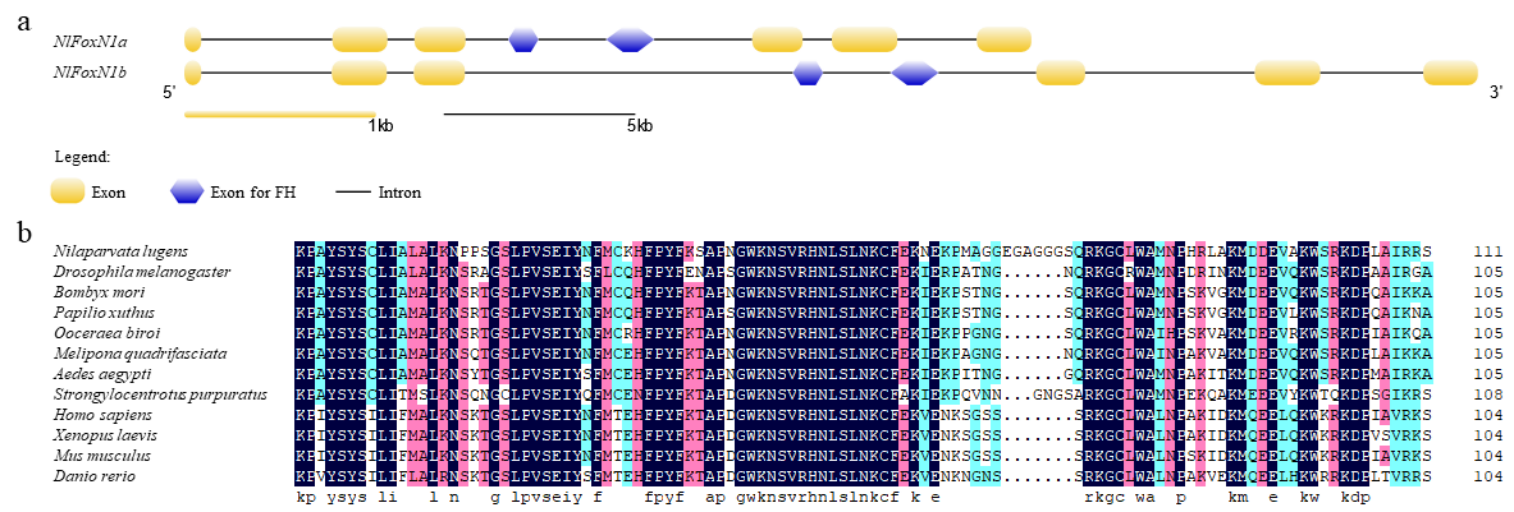

Figure 1. Sequence analysis of NlFoxN1. (a) Exon-intron analysis of NlFoxN1 was carried out by GSDS2.0. (b) Alignment of amino acid sequences of forkhead domain (FHD) from 12 species was carried out by DNAMAN.

\subsection{Temporal and Spatial Expression Patterns of NlFoxN1}

The spatial and temporal expression patterns of NlFoxN1 were assessed by real-time quantitative PCR (qPCR). To explore the developmental expression patterns, total RNA was extracted from various developmental samples containing all life stages of BPHs (eggs, nymphs in five different instars, female adults, and male adults). The results showed that NlFoxN1 was highly expressed in newly laid eggs, 
which was the early stage of embryogenesis, indicating that NlFoxN1 might play an important role in the embryonic development of fertilized eggs. Compared to that in males, the transcripts of NlFoxN1 in females were maintained at a relatively higher level (Figure 2a). The tissue samples used for the tissue-specific expression patterns were extracted from random adults including gut, salivary gland, fat body, integument, testis, and ovary. NlFoxN1 was mainly expressed in the ovaries, suggesting that eggs have begun to express NIFoxN1 in the maternal stage (Figure 2b).

a

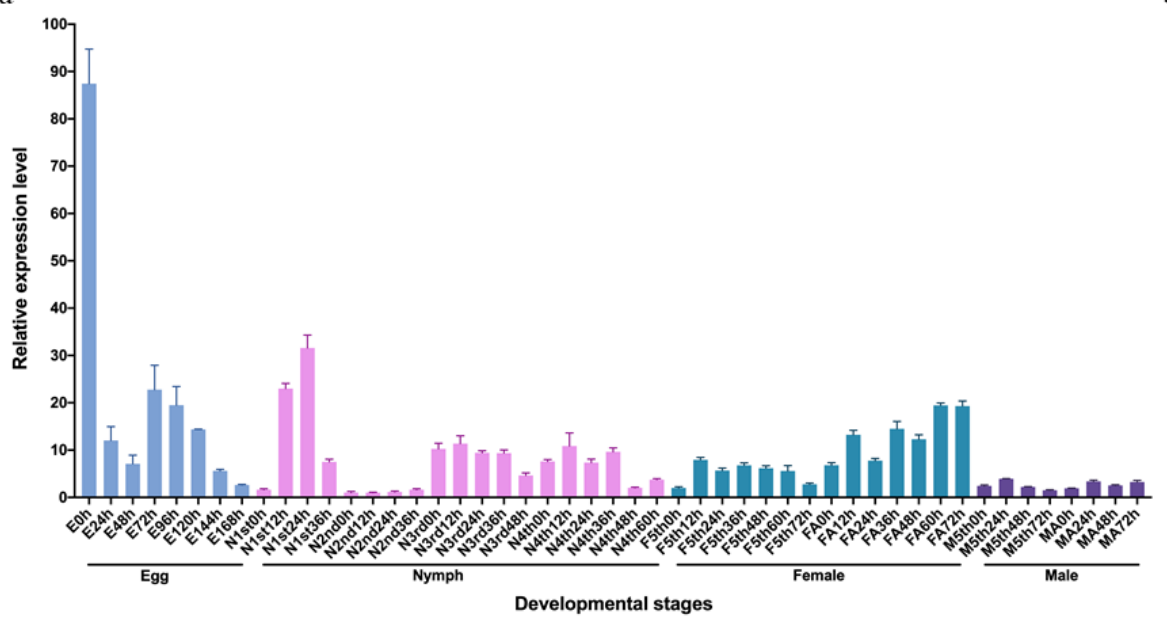

b

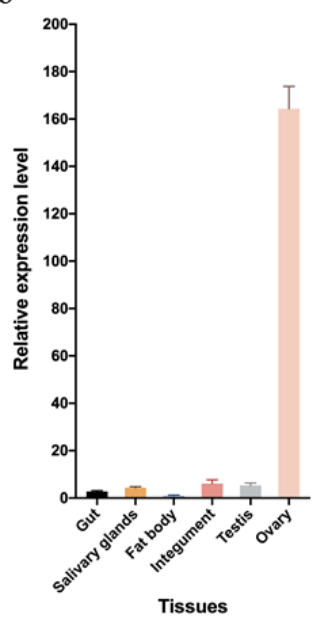

Figure 2. Temporal and spatial expression patterns of NIFoxN1. (a) Expression patterns in different development stages. E: egg; N: nymphs regardless of gender; F: female; FA: female adult; M: male; MA: male adult. (b) Expression patterns in different tissues. Developmental samples were extracted from whole insects at all life stages of BPHs ( $n=200$ eggs, $n=30$ nymphs and adults). Samples were collected every $12 \mathrm{~h}$ or $24 \mathrm{~h}$ from the very beginning of each stage. Tissue samples were dissected from 50 random adults $48-72 \mathrm{~h}$ after eclosion. Nl18S was used as an internal control gene for qPCR. Mean \pm standard error of the mean (s.e.m.) from three experiments.

\subsection{NlFoxN1 Was Indispensable for Embryogenesis}

To determine the function of the NIFoxN1, we conducted RNAi experiments. To avoid off-target effects, RNAi experiments were replicated by choosing two non-overlapping regions as targets. The qPCR analysis revealed that each dsRNA efficiently suppressed the transcript levels of their target genes (Table S1).

Since NlFoxN1 was highly expressed in the eggs, we conducted the RNAi experiments on newly emerged female adults, at the time when the ovaries were not fully developed, to observe the phenotypes of egg production and embryonic development in the next generation. The ovaries developed normally after injection, and there was no significant difference in egg production compared with the dsGFP-treated group, but the hatchability of the eggs was seriously affected (Figure 3a). The eggs without expression of NlFoxN1 could not develop normally. The red eyespots (compound eye buds) that should appear about 4 days after egg production did not appear, without exception, indicating that embryonic development is terminated before the stage of embryonic movement (Figure $3 b$ ). 
a

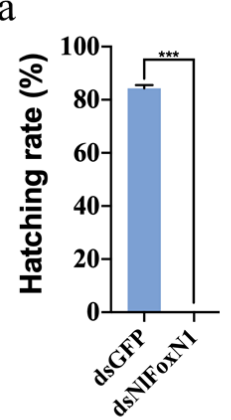

b

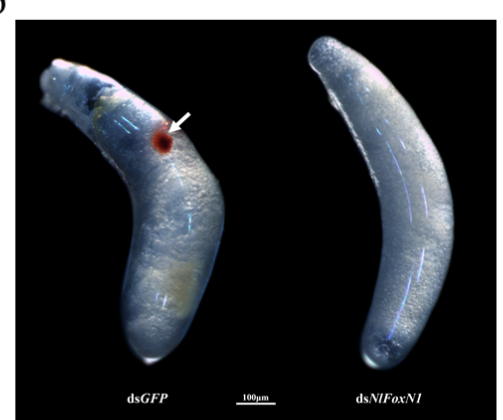

$\mathrm{c}$

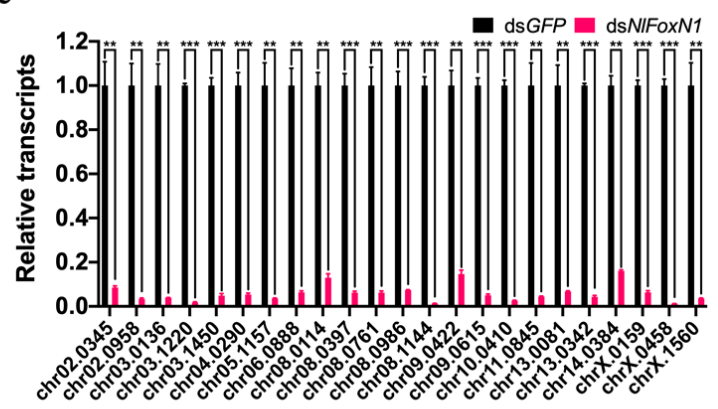

d
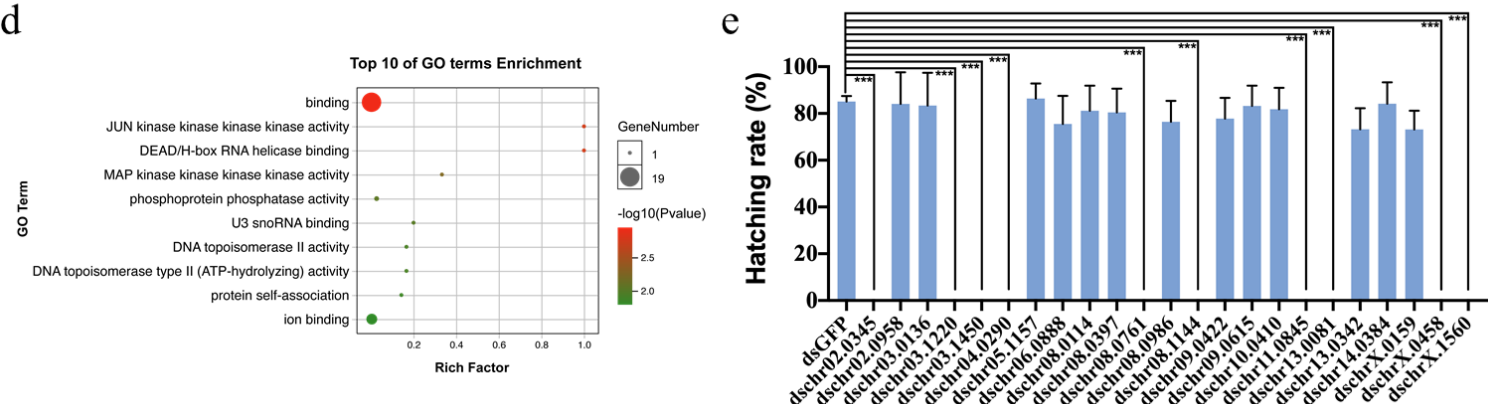

f

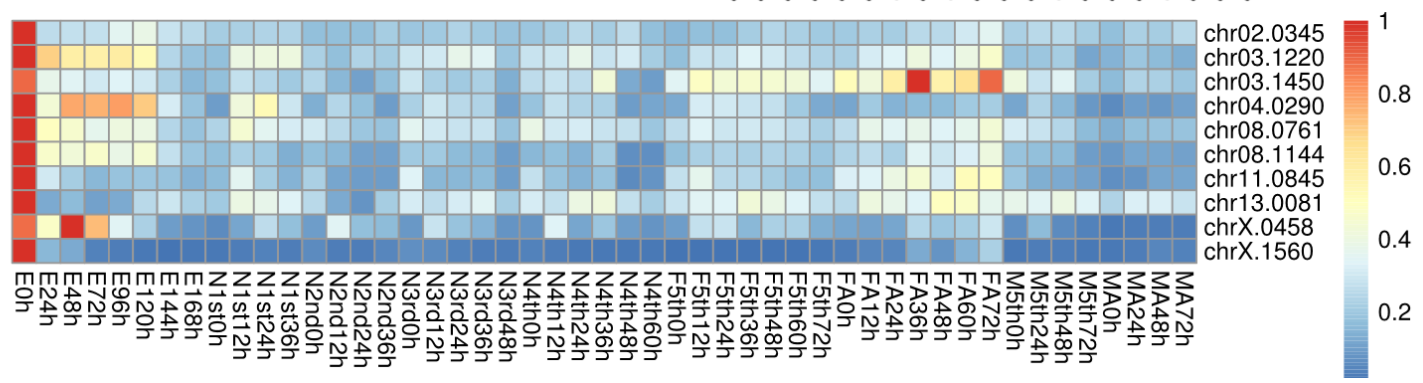

Figure 3. NlFoxN1 was indispensable for embryogenesis. (a) The hatching rate of the eggs after knockdown of NlFoxN1. (b) The lethal phenotypes of eggs after knockdown of NIFoxN1. The red eyespot was marked by the white arrow. (c) qPCR verification of the differentially expressed genes (DEGs). (d) Gene Ontology (GO) term enrichment analysis of the 23 verified DEGs in eggs. The GO enrichment study was performed using OmicShare tools. (e) The hatching rates of the eggs after knockdown of the 23 verified DEGs, respectively. (f) The temporal expression patterns of the ten potential targets. Heatmap was plotted using pheatmap R package based on the qPCR data from three biological replicates. dsRNA (50 ng per insect; $n=100$ ) was injected into newly emerged female adults (within two hours). dsGFP was injected as a negative control for the nonspecific effects of dsRNA. Mean \pm s.e.m. from three experiments. ${ }^{* *} p<0.01$ and ${ }^{* *} p<0.001$ (Student's $t$-test), difference from dsGFP.

\subsection{Potential Targets of NIFoxN1 in the Eggs}

Large scale RNAi and RNA-seq were used to reveal potential target genes of NlFoxN1 in the eggs. A total of 63 differentially expressed genes (DEGs) were identified by transcriptome sequencing, among which, 59 genes were significantly downregulated after knockdown of NlFoxN1 in the newly laid eggs. qPCR assays confirmed that 23 of the DEGs were downregulated by NlFoxN1 including several transcription factors, and the four upregulated genes were proven insignificant (Figure 3c). To extrapolate the biological processes of the DEGs, we performed Gene Ontology (GO) term enrichment analysis. We found that a total of $82.6 \%$ of the 23 downregulated genes were significantly enriched in binding activities (GO:0005488), which is defined as interaction of a molecule with one or more specific sites on another molecule, including protein and nucleic acid binding activities (Figure 3d).

To determine the functions of the 23 downregulated genes, dsRNAs targeting each of these genes were injected into newly emerged female adults (within two hours), respectively. The RNAi results 
indicated that ten genes were indispensable to embryogenesis and knockdown of any of these ten genes would therefore result in a zero-hatching rate (Figure 3e). Furthermore, these ten genes had similar expression patterns to NlFoxN1, highly expressed in the newly laid eggs (Figure 3f). These results suggested that these ten genes were the potential target genes of NlFoxN1, and therefore accounted for the lethal effect of NlFoxN1 knockdown in early egg stages.

\subsection{NlFoxN1 Regulated Keratin Genes to Affect Molting Process}

In addition to the high expression levels in eggs and adults, NlFoxN1 also had a certain level of expression in nymphs. To investigate the possible functions of NlFoxN1 in nymphs, dsRNA was injected into newly emerged 4 th and 5th instar nymphs (within two hours), respectively.

Injection of dsRNA for NIFoxN1 in early fifth-instar nymphs led to a lethal phenotype, the death rate reached almost 100\% (Figure 4a). All dead BPHs exhibited the same phenotype, they failed to shed their old cuticles and died quickly during nymph-to-adult molting (Figure $4 \mathrm{~b}$ ). The same phenotype was found between 4 th to 5 th nymph molting after injecting NlFoxN1 dsRNA into early fourth-instar nymphs (Figure 4c). To investigate the changes in gene expression resulting from depletion of NlFoxN1, we made transcriptomic analysis using RNA-seq. A total of 248 up- and 652 downregulated genes were detected in the NlFoxN1-RNAi nymphs (4th $48 \mathrm{~h}$ ). The GO terms of the DEGs were significantly enriched in the chitin-based cuticle (GO:0005214) for both biological process and molecular function categories, while for cellular components, the enriched GO terms were related to the extracellular region (GO:0005576) (Figure 5).

a

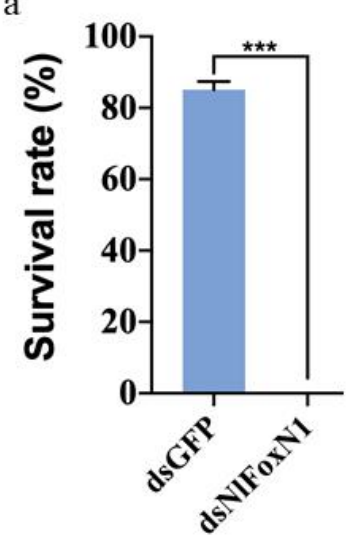

b

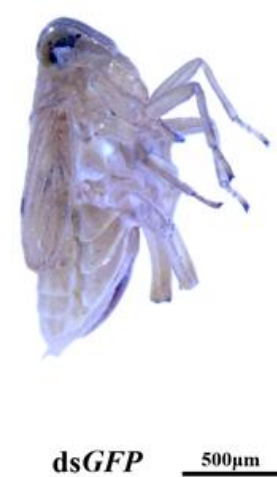

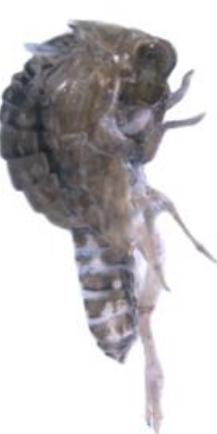

dsNIFoxN1

c

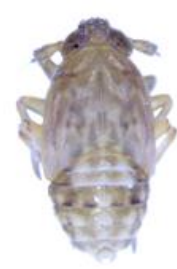

dsGFP

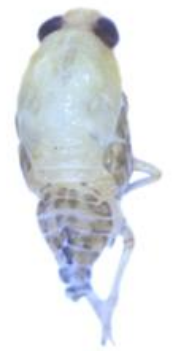

dsNIFoxN1

Figure 4. Knockdown of NlFoxN1 in nymphs affected the molting process. (a) The survival rate after NlFoxN1-RNAi in the fifth instar. (b,c) The lethal phenotypes after injection of dsRNA for NlFoxN1 in the fifth instar (b) and the fourth instar (c). dsRNA (50 ng per insect; $n=100$ ) was injected into BPHs at the very beginning of each instar. dsGFP was injected as a negative control for the nonspecific effects of dsRNA. Mean \pm s.e.m. from three experiments. ${ }^{* * *} p<0.001$ (Student's $t$-test), difference from dsGFP.

Among these DEGs, three keratin genes encoding type I cytoskeletal keratin 9 (Krt9), type I cytoskeletal keratin 10 (Krt10), and type II cytoskeletal keratin 1 (Krt1) were downregulated by dsNlFoxN1. The keratin genes were highly expressed in the integument (cuticles) (Figure 6a). The qPCR results further confirmed that the three keratin genes were significantly downregulated after the injection of dsNlFoxN1 in 4th instar nymphs (Figure 6b). To determine the relationship between the keratin genes and the high mortality, microinjection experiments were performed with dsRNAs targeting NlKrt1, $N l K r t 9$, and NlKrt10, respectively. The RNAi results showed that knockdown of NlKrt9 resulted in the same phenotypes with NlFoxN1. The NlKrt9-RNAi nymphs had a high mortality (Figure 6c) and molting dysfunction (Figure 6d). However, injection of the dsRNA for NlKrt1 or NlKrt10 did not result in any abnormal molting or other observable morphological or inter-structural abnormalities. 


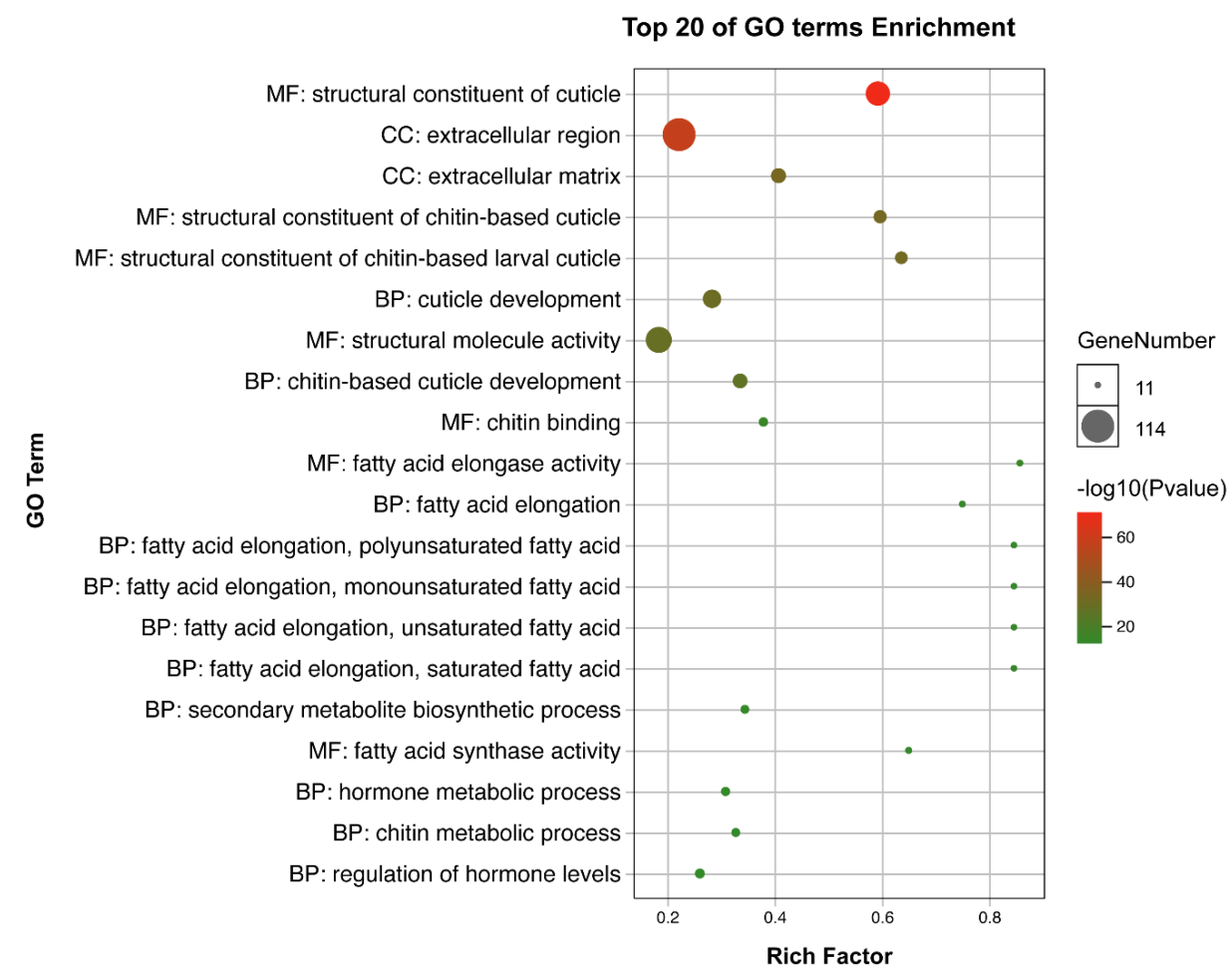

Figure 5. The GO enrichment analysis of the DEGs in nymphs. The GO enrichment study was performed using OmicShare tools. MF: molecular function, CC: cellular component, bp: biological process.

a

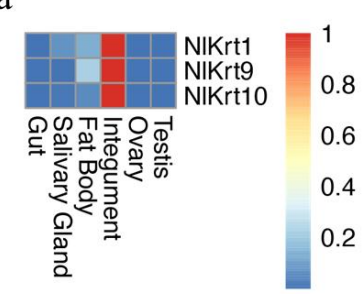

$\mathrm{d}$

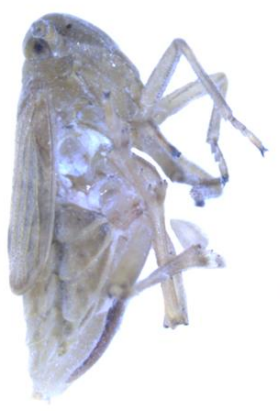

ds $G F P$ b
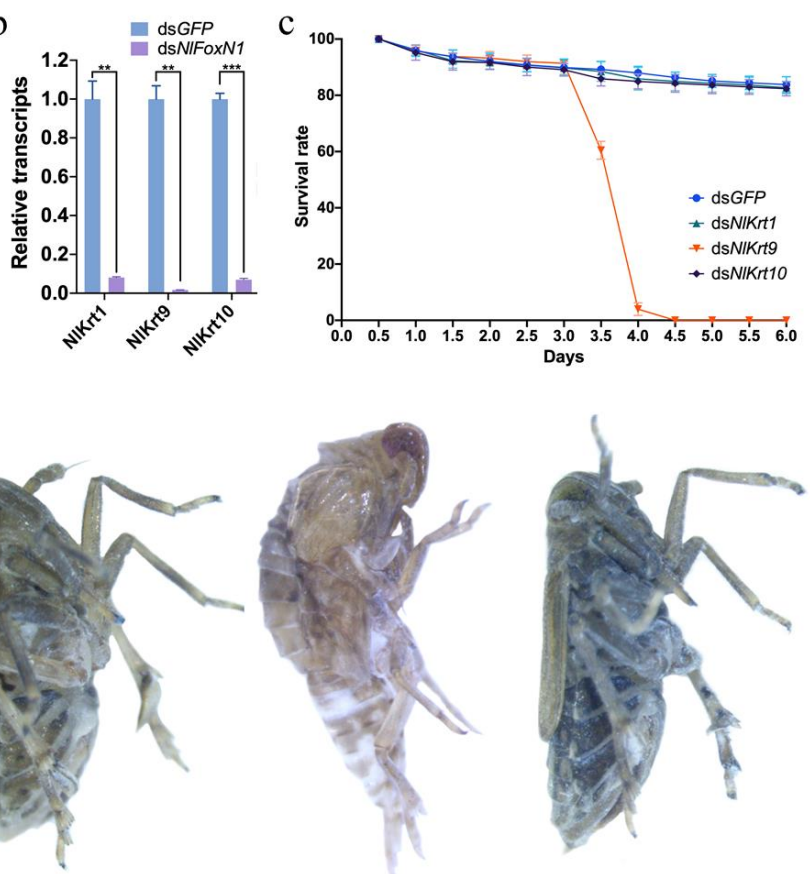

dsNlKrt10

Figure 6. NIFoxN1 regulated keratin genes to affect molting process. (a) Tissue-specific expression patterns of the keratin genes. (b) Knockdown of NlFoxN1 decreased expression levels of keratin genes in the nymphs. (c) The survival rates after knockdown of the keratin genes in the fifth instar. (d) The phenotypes after injection of dsRNA for keratin genes in the fifth instar. dsRNA (50 ng per insect; $n=100$ ) was injected into BPHs at the very beginning of the fifth instar. dsGFP was injected as negative control for the nonspecific effects of dsRNA. Mean \pm s.e.m. from three experiments. ${ }^{* *} p<0.01$ and ${ }^{* * *} p<0.001$ (Student's $t$-test), difference from dsGFP. 


\section{Discussion}

The results of the RNAi experiments indicated that NlFoxN1 exhibited pleiotropism; not only affecting the egg hatching rate in eggs, but also playing an important role in molting in nymphs. RNA-seq has become a powerful tool to investigate transcriptome profiling using deep-sequencing technologies [15]. To investigate DEGs of NlFoxN1 in different stages, we used RNA-seq to profile the transcriptome after RNAi in eggs and nymphs, respectively. The DEGs verified by qPCR and RNAi assays were defined as the potential target genes.

NIFoxN1 was highly expressed in the very beginning of the eggs and was indispensable to embryonic development, probably acting as a "pioneer" factor which is involved in the regulation of cell differentiation in the early stages of embryonic development [16]. Pioneer factors are able to access their target sequence by interacting with condensed chromatin and open the way for the expression of formerly silent important transcription factors, thus making the regulated genes available for activation [17]. Such activity might explain that potential target genes of NlFoxN1 in the newly laid eggs contained multiple transcription factors. NlFoxN1 activated the ten potential target genes which had the similar expression patterns and functions with NlFoxN1 to participate in embryogenesis in eggs.

In nymphs, cuticles are a complex composite material, made of chitin filaments embedded in cuticular proteins [18]. It provides structural and mechanical support by serving functionally as both skin and skeleton [19]. There are obvious keratinization defects in the epidermis and nails of nude mice, and Foxn1 malfunction can result in the suppression of keratin genes [20]. Our RNA-seq results indicated that three N. lugens keratin genes (NlKrt1, NlKrt9, and NlKrt10) were downstream genes of NlFoxN1. Moreover, knockdown of NlKrt9 results in the same phenotypes with that of NlFoxN1-RNAi; defects in molting with a high mortality. Krt9 is an intermediate filament (IF) protein, specifically expressed in the suprabasal layers of the palmoplantar epidermis in humans [21]. Dominant-negative mutations in the Krt9 gene cause epidermolytic palmoplantar keratoderma, a rare skin disorder [22]. The IF framework that exists in insect cells is similar to the IF framework that exists in mammalian cells [23]. The evidence strongly suggests that NlFoxN1 participates in the formation of the IF framework by regulating the expression of $N l K r t 9$, thereby influencing the molting process.

The pleiotropism of FoxN1 in the mammals is controlled by alternative splicing with two different first exons [12]. NlFoxN1 had only one splicing but two duplicated genes in the N. lugens genome, indicating that the pleiotropic mechanism of NIFoxN1 was different from that in the mammals. However, whether the pleiotropic mechanism of NlFoxN1 is regulated by duplicated genes requires further study.

The evolutionary conservation of the crucial DNA-binding domain between orthologous members of the Fox gene family is remarkable [24]. Our analysis showed that the sequences of FoxN1 are highly conserved both in vertebrates and invertebrates. The Drosophila homologue of FoxN1 is jumeaux [25], which is required for normal eye and wing morphogenesis and appears to act in the modification of chromatin structure [26]. In Aedes aegypti, FoxN1 is strongly expressed in the ovaries and is important in regulating mosquito reproduction [27]. This means that the roles of FoxN1 in embryogenesis and cuticles also seem somewhat conserved in insects.

Altogether, NlFoxN1 regulated ten potential target genes including several transcription factors to initiate embryogenesis in the eggs, and regulated keratin genes to maintain the homeostasis of nymph cuticles, thus demonstrating the pleiotropic functions of NlFoxN1. These findings may furthermore stimulate the design and development of novel insecticides which could control both current and next generation populations.

\section{Materials and Methods}

\subsection{Insects}

The insects used in this study were obtained from local rice fields at Zhejiang University, Hangzhou, Zhejiang, China. The insects were reared on fresh rice seedlings (Xiushui 134) at $25 \pm 1^{\circ} \mathrm{C}$ and $60-70 \%$ relative humidity under a $16 \mathrm{~h} / 8 \mathrm{~h}$ (light/dark) photoperiod [28]. 


\subsection{Gene Identification and Sequence Analyses}

Nilaparvata lugens genomic [29] and transcriptomic databases were screened for the gene encoding FoxN1 against the amino acid sequences from Drosophila melanogaster, Mus musculus, and Homo sapiens, which were obtained from GenBank. The full-length cDNA sequence was obtained from transcriptomic databases and confirmed by RT-PCR. The gene structure display server program (GSDS2.0) was employed to identify gene structures [30]. Multiple sequence alignments were performed using the ClustalX program [31]. The primers used here are shown in Table S1.

\subsection{Expression Pattern Analysis}

Total RNA from whole insects at various developmental stages or tissue samples was isolated using a TRIzol Total RNA Isolation Kit (Takara, Kyoto, Japan). Developmental samples were collected from different stages of BPHs $(n=15-20)$, including 8 egg samples, 30 nymph samples, 7 female adult samples, and 4 male adult samples. Similarly, various tissue samples, including integument, gut, fat body, salivary gland, testis, and ovary were dissected from random female adults $48-72 \mathrm{~h}$ after adult emergence. Three biological replications were performed for each developmental and tissue sample. Each biological replicate consisted of $N$. lugens eggs, nymphs, adults or specific tissue samples. To investigate the developmental and tissue-specific expression patterns, qPCR was conducted using ChamQ SYBR Color qPCR Master Mix (Vazyme Biotech Co.,Ltd). The pairs of gene-specific primers were designed using the Primer Premier 6 program (Table S1). The N. lugens housekeeping gene for 18S ribosomal RNA (Nl18S) (GenBank accessionnumber JN662398.1) was used as an internal control.

\subsection{RNAi Effects on N. lugens}

The double-stranded RNA (dsRNA) was synthesized using a T7 High Yield RNA Transcription Kit (Vazyme Biotech Co.,Ltd). A unique region of each gene was chosen as a template for dsRNA synthesis. The primers used for the dsRNA synthesis can be found in Table S1. The dsRNA for GFP was used as a negative control for the nonspecific effects of dsRNA. Microinjection of planthoppers with dsRNA was carried out according to a previously reported method [32]. One hundred and fifty insects were used for each gene treatment, and each treatment was conducted in triplicate. Each insect was injected with $10 \mu \mathrm{L}$ of dsRNA, at a concentration of $5 \mathrm{ng} / \mu \mathrm{L}$. Samples were collected from a set of 6-10 insects to evaluate the RNAi effects of each gene. A second, non-overlapping region was selected for dsRNA synthesis to overcome possible off-target effects.

\subsection{Differential Expression Analysis Using RNA-seq}

The newly emerged female adults were injected with dsRNA, paired with normal male adults, and kept for three days on fresh rice seedlings until they reached sexual maturity. Approximately 500 eggs newly laid on rice stems were carefully dissected for total RNA extraction. Thirty individuals, $48 \mathrm{~h}$ after injecting dsRNA in the fourth instar nymphs, were homogenized for total RNA extraction. The dsRNA for GFP was used as a negative control for the nonspecific effects of the dsRNA. Each treatment involved three sets of biological replicates. The cDNA library preparation and Illumina sequencing were performed using Annoroad (Beijing, China).

The clean reads were aligned to the reference genome using HISAT2 [33]. The low-quality alignments were filtered with SAMtools [34]. TPM expression values were calculated using featureCounts for genes [35]. Differential expression analysis was carried out using the DESeq2 package [36]. The differentially expressed genes needed to meet the following conditions: false discovery rate (FDR) $<0.05$ and absolute value of the $\log 2$ ratio $>2$.

Supplementary Materials: Supplementary materials can be found at http://www.mdpi.com/1422-0067/21/12/ 4222/s1. Table S1. The primers and RNAi efficiency in this study. 
Author Contributions: Conceptualization, C.-X.Z.; data curation, Y.-X.Y.; investigation, Y.-X.Y.; supervision, C.-X.Z.; visualization, Y.-X.Y.; writing-original draft, Y.-X.Y.; writing-review and editing, C.-X.Z. All authors read and agreed to the published version of the manuscript.

Funding: This research was funded by the National Natural Science Foundation of China (31900361, 31871954 and 31630057) and China Postdoctoral Science Foundation (2018M642453 and 2019T120523).

Conflicts of Interest: The authors declare no conflict of interest.

\section{References}

1. Kaestner, K.H.; Knochel, W.; Martinez, D.E. Unified nomenclature for the winged helix/forkhead transcription factors. Genes Dev. 2000, 14, 142-146.

2. Weigel, D.; Jäckle, H. The fork head domain: A novel DNA binding motif of eukaryotic transcription factors? Cell 1990, 63, 455-456. [CrossRef]

3. Schorpp, M.; Hofmann, M.; Dear, T.N.; Boehm, T. Characterization of mouse and human nude genes. Immunogenetics 1997, 46, 509-515. [CrossRef]

4. Schlake, T.; Schorpp, M.; Nehls, M.; Boehm, T. The nude gene encodes a sequence-specific DNA binding protein with homologs in organisms that lack an anticipatory immune system. Proc. Natl. Acad. Sci. USA 1997, 94, 3842-3847. [CrossRef] [PubMed]

5. David, L.; David, M.P.; Janice, L.B. Association between Mouse nude Gene Expression and the Initiation of Epithelial Terminal Differentiation. Dev. Biol. 1999, 208, 362-374.

6. Brissette, J.L.; Li, J.; Kamimura, J.; Lee, D.; Dotto, G.P. The product of the mouse nude locus, Whn, regulates the balance between epithelial cell growth and differentiation. Genes Dev. 1996, 10, 2212-2221. [CrossRef] [PubMed]

7. Palamaro, L.; Romano, R.; Fusco, A.; Giardino, G.; Gallo, V.; Pignata, C. FOXN1 in Organ Development and Human Diseases. Int. Rev. Immunol. 2014, 33, 83-93. [CrossRef] [PubMed]

8. Grabowska, A.I.; Wilanowski, T. FOXN1 Transcription Factor in Epithelial Growth and Wound Healing. Mol. Cell Boil. 2017, 37. [CrossRef] [PubMed]

9. Nehls, M.; Kyewski, B.; Messerle, M.; Waldschütz, R.; Schuddekopf, K.; Smith, A.J.H.; Boehm, T. Two Genetically Separable Steps in the Differentiation of Thymic Epithelium. Science 1996, 272, 886-889. [CrossRef]

10. Romano, R.; Palamaro, L.; Fusco, A.; Giardino, G.; Gallo, V.; Del Vecchio, L.; Pignata, C. FOXN1: A mster regulator gene of thymic epithelial development program. Front. Immunol. 2013, 4. [CrossRef]

11. Rota, I.A.; Dhalla, F. FOXN1 deficient nude severe combined immunodeficiency. Orphanet J. Rare Dis. $2017,12$. [CrossRef] [PubMed]

12. Schlake, T. The nude gene and the skin. Exp. Dermatol. 2001, 10, 293-304. [CrossRef] [PubMed]

13. Lam, E.W.-F.; Brosens, J.J.; Gomes, A.R.; Koo, C.Y. Forkhead box proteins: Tuning forks for transcriptional harmony. Nat. Rev. Cancer 2013, 13, 482-495. [CrossRef] [PubMed]

14. Ab Ghaffar, M.B.; Pritchard, J.; Ford-Lloyd, B. Brown Planthopper (N. lugens Stal) Feeding Behaviour on Rice Germplasm as an Indicator of Resistance. PLoS ONE 2011, 6. [CrossRef] [PubMed]

15. Wang, Z.; Gerstein, M.; Snyder, M. RNA-Seq: A revolutionary tool for transcriptomics. Nat. Rev. Genet. 2009, 10, 57-63. [CrossRef] [PubMed]

16. Vaidya, H.J.; Leon, A.B.; Blackburn, C.C. FOXN1 in thymus organogenesis and development. Eur. J. Immunol. 2016, 46, 1826-1837. [CrossRef]

17. Zaret, K.S.; Carroll, J.S. Pioneer transcription factors: Establishing competence for gene expression. Genes Dev. 2011, 25, 2227-2241. [CrossRef]

18. Andersen, S.O.; Hojrup, P.; Roepstorff, P. Insect cuticular proteins. Insect Biochem. Mol. Boil. 1995, 25, $153-176$. [CrossRef]

19. Hamodrakas, S.; Willis, J.H.; Iconomidou, V.A. A structural model of the chitin-binding domain of cuticle proteins. Insect Biochem. Mol. Boil. 2002, 32, 1577-1583. [CrossRef]

20. Mecklenburg, L.; Nakamura, M.; Sundberg, J.P.; Paus, R. The Nude Mouse Skin Phenotype: The Role of Foxn1 in Hair Follicle Development and Cycling. Exp. Mol. Pathol. 2001, 71, 171-178. [CrossRef]

21. Fu, D.J.; Thomson, C.; Lunny, D.P.; Dopping-Hepenstal, P.J.; McGrath, J.A.; Smith, F.J.; McLean, W.H.I.; Pedrioli, D.L. Keratin 9 Is Required for the Structural Integrity and Terminal Differentiation of the Palmoplantar Epidermis. J. Investig. Dermatol. 2013, 134, 754-763. [CrossRef] [PubMed] 
22. Smith, F. The molecular genetics of keratin disorders. Am. J. Clin. Dermatol. 2003, 4, 347-364. [CrossRef] [PubMed]

23. Chen, X.; Yu, Z.; Chen, Q. Detection of keratin in insect cells. J. Histochem. Cytochem. 1996, 5, $214-218$.

24. Hannenhalli, S.; Kaestner, K.H. The evolution of Fox genes and their role in development and disease. Nat. Rev. Genet. 2009, 10, 233-240. [CrossRef]

25. Cheah, P.Y.; Chia, W.; Yang, X. Jumeaux, a novel Drosophila winged-helix family protein, is required for generating asymmetric sibling neuronal cell fates. Development 2000, 127, 3325-3335.

26. Lee, H.-H.; Frasch, M. Survey of forkhead domain encoding genes in the Drosophila genome: Classification and embryonic expression patterns. Dev. Dyn. 2004, 229, 357-366. [CrossRef] [PubMed]

27. Hansen, I.A.; Sieglaff, U.H.; Munro, J.; Shiao, S.-H.; Cruz, J.; Lee, I.W.; Heraty, J.M.; Raikhel, A.S. Forkhead transcription factors regulate mosquito reproduction. Insect Biochem. Mol. Boil. 2007, 37, 985-997. [CrossRef]

28. Ye, Y.-X.; Pan, P.-L.; Kang, D.; Lu, J.-B.; Zhang, C.-X. The multicopper oxidase gene family in the brown planthopper, Nilaparvata lugens. Insect Biochem. Mol. Boil. 2015, 63, 124-132. [CrossRef]

29. Xue, J.; Zhou, X.; Zhang, C.-X.; Yu, L.-L.; Fan, H.-W.; Wang, Z.; Xu, H.-J.; Xi, Y.; Zhu, Z.-R.; Zhou, W.; et al. Genomes of the rice pest brown planthopper and its endosymbionts reveal complex complementary contributions for host adaptation. Genome Biol. 2014, 15. [CrossRef]

30. Hu, B.; Jin, J.; Guo, A.-Y.; Zhang, H.; Luo, J.; Gao, G. GSDS 2.0: An upgraded gene feature visualization server. Bioinformatics 2014, 31, 1296-1297. [CrossRef]

31. Larkin, M.; Blackshields, G.; Brown, N.; Chenna, R.; Mcgettigan, P.; McWilliam, H.; Valentin, F.; Wallace, I.; Wilm, A.; López, R.; et al. Clustal W and Clustal X version 2.0. Bioinformatics 2007, 23, 2947-2948. [CrossRef] [PubMed]

32. Xu, H.-J.; Xue, J.; Lu, B.; Zhang, X.-C.; Zhuo, J.-C.; He, S.-F.; Ma, X.-F.; Jiang, Y.-Q.; Fan, H.-W.; Xu, J.-Y.; et al. Two insulin receptors determine alternative wing morphs in planthoppers. Nature 2015, 519, 464-467. [CrossRef] [PubMed]

33. Pertea, M.; Kim, D.; Pertea, G.M.; Leek, J.T.; Salzberg, S.L. Transcript-level expression analysis of RNA-seq experiments with HISAT, StringTie and Ballgown. Nat. Protoc. 2016, 11, 1650-1667. [CrossRef] [PubMed]

34. Li, H.; Handsaker, B.; Wysoker, A.; Fennell, T.; Ruan, J.; Homer, N.; Marth, G.; Abecasis, G.R.; Durbin, R.; 1000 Genome Project Data Processing Subgroup. The Sequence Alignment/Map format and SAMtools. Bioinformatics 2009, 25, 2078-2079. [CrossRef]

35. Liao, Y.; Smyth, G.K.; Shi, W. featureCounts: An efficient general purpose program for assigning sequence reads to genomic features. Bioinformatics 2013, 30, 923-930. [CrossRef]

36. Love, M.I.; Huber, W.; Anders, S. Moderated estimation of fold change and dispersion for RNA-seq data with DESeq2. Genome Biol. 2014, 15. [CrossRef] 